\title{
Platelet Function Closure Time Measurement
}

National Cancer Institute

\section{Source}

National Cancer Institute. Platelet Function Closure Time Measurement. NCI Thesaurus.

Code C111295.

The determination of the collagen closure time, as a surrog ate of platelet function, present in a sample. 